\title{
Analysis of Knowledge Management Experiences in Spanish Public Administration
}

\author{
Alicia García-Holgado \\ GRIAL Research Group, \\ Research Institute for Educational \\ Sciences, \\ University of Salamanca \\ Paseo de Canalejas 169, 37008 \\ Salamanca, Spain \\ (+34) 923294500 ext. 3433 \\ aliciagh@usal.es
}

\author{
Juan Cruz-Benito \\ GRIAL Research Group, \\ Research Institute for Educational \\ Sciences, \\ University of Salamanca \\ Paseo de Canalejas 169, 37008 \\ Salamanca, Spain \\ (+34) 923294500 ext. 3433 \\ juancb@usal.es
}

\author{
Francisco J. García-Peñalvo \\ GRIAL Research Group, \\ Research Institute for Educational \\ Sciences, \\ University of Salamanca \\ Paseo de Canalejas 169, 37008 \\ Salamanca, Spain \\ (+34) 923294500 ext. 3433 \\ fgarcia@usal.es
}

\begin{abstract}
Under the development of the Digital Society and with the aim of achieving a true transition from the Information Society to the Knowledge Society, ICTs play a capital role in educational and knowledge management processes in any kind of entity, from Small and Medium-sized Enterprises to the Public Administration. The Spanish Public Administration is composed by a huge amount of the heterogeneous public organisms that range from research centres to public companies. The purpose of this paper is to provide an analysis about the knowledge management in the Spanish Public Administration through several real study cases developed in different public organisms. The analysis shows how the case studies follow the Suricata Architecture in order to identify success factors to replicate them in other public contexts.
\end{abstract}

\section{Categories and Subject Descriptors}

H.1.2 [Models and Principles]: User/Machine Systems - Human factors. H.4.2 [Information Systems Applications]: Type of Systems. K.3.1 [Computers and Education]: Computer uses in education.

\section{General Terms}

Management, Documentation, Experimentation, Humán Factors.

\section{Keywords}

Knowledge Management, Knowledge Society, Public Administration, Analysis

\section{INTRODUCTION}

In the past few years, the previously known as Information Society has suffered a significant evolution that has given way to the current Knowledge Society. The issues focused on technology as main element in the Information Society has provided the development of the Knowledge Society in which core element is the ability to identify, produce, process, transform, disseminate and use the information to build and apply knowledge for human development [20].

The Knowledge Society is a Learning Society, where the learning is the key factor in order to persons, business, regions and countries achieve success [15].

In this context, the knowledge management emerges as a sustainable competitive advantage [14] so the organizations expend part of their resources on building their capacity to share, create and apply new knowledge continuously over time [8].

The learning is a base element inside any kind of organization, regardless of its size or the activity that it develops. From the Small and Medium Enterprises (SME) to the institutions such Universities or Public Administration (PA) include mechanisms that allow the knowledge management inside their processes.

The purpose of this work is to present the analysis about the knowledge management in real contexts related to the Spanish Public Administration, in order to highlight the successes or failures of each analysed case study.

The following sections present the context in which the selected case studies have been developed (2), the analysed case studies (3), the obtained results (4) and finally the main conclusions of this work.

\section{CONTEXT}

According to Nonaka and Takeuchi [14] there are two types of knowledge depending on their nature, explicit knowledge and tacit knowledge. The explicit knowledge is a structured, storable and easy to communicate knowledge, which involves a commitment by a particular person. Regarding to the tacit knowledge, it is hard to formalize and difficult to capture and communicate to others because it is conditioned by intangible factors such as socio-cultural context.

The knowledge management processes should be able to support the transfer of tacit knowledge to explicit knowledge, due to this, Nonaka and Takeuchi [14] define the cycles model of knowledge creation, also called SECI model, which covers four modes of knowledge creation: internalization, socialization, externalization and combination.

Regardless of their nature, organizations have the knowledge management as main goal to achieve success [21]. At the present 
technological context, the knowledge management systems provide the required tools to support processes and facilitate the access and reuse of knowledge, well as promote knowledge growth, communication, preservation and sharing [13]. The technological ecosystem concept appears when the organization wants to ensure that the provided set of tools and services have a high integration degree and a strong evolving component in all its aspects [5].

The knowledge management inside an organization depends on a large number of factors both internal (employees profile, workflows, etc.) and external (cultural contexts, market, etc.), which have influence directly in the technological ecosystem that supports such management [6], in such a way that each ecosystem is unique.

The analysis of knowledge management systems based on technological ecosystems allows detect problems that arise when these kind of technological solutions are implemented, regardless the contexts where they are used. Authors have presented in previous works $[6 ; 7]$ solutions with a strong engineering component which aim to establish the base to develop ecosystems with a high integration and cohesion degree, and with capability to evolve at the same time that organizations and person.

The Public Administration provides a suitable context to analyse the knowledge management, due to it is composed by a heterogeneous set of public organisms that range from research centres to different types of public companies. This provide an overview of how knowledge is created, transformed, distributed and evolves in rich and heterogeneous public contexts, so the analysis results will be able to applied to improve the technological ecosystems in any scope through the know-how extension and the wisdom obtained in such contexts about the problem domain [17].

\section{KNOWLEDGE MANAGEMENT EXPERIENCES}

In order to illustrate different possibilities of knowledge management inside the Spanish Public Administration, this work selects two representative case studies that provide an introduction to the presented contents. First, this work presents the case study of the National Institute of Public Administration (Instituto Nacional de Administración Pública, TNAP in Spanish) (http://www.inap.es), whose main goal is improvement the information flows and the informal learning between employees that compose the Spanish Public Administration. Meanwhile, the second case study is focused on the study and analysis of the management and exploitation of sense knowledge, from a social and economic point of view, in the complex scope of the University and the employability of graduates, describing the expertise and technology developed by the Observatory for University Employability and Employment (Observatorio de Empleabilidad y Empleo Universitarios, OEEU in Spanish) (http://oeeu.org) led by the UNESCO Chair in University Management and Policy at the Technical University of Madrid (UPM).

\subsection{National Institute of Public Administration}

The INAP is an autonomous body within the national Government of Spain, attached to the Department of Finance and Public Administration. The Institute possesses a huge experience in knowledge management inside the Public Administration. We can find the following activities between its main tasks: training and professional improvement for public employees; recruitment into the Civil Service; and promoting research and studies regarding government and the different levels on Public Administration from an interdisciplinary perspective.

The technological ecosystem of INAP is composed by a large number of components oriented to cover the different knowledge management needs both Institute inside and outside. In the scope of this work, there are three main components: the communities of practices, named INAP Social (http://social.inap.es); the Knowledge Bank or BCI (Banco de Conocimiento, in Spanish) (http://bci.inap.es); and the courses repository, Compartir (http://compartir.inap.es). The social and knowledge ecosystem of INAP is fully described in the book Conocimiento transformador y talento público. El caso del INAP [2].

The architecture of the technological ecosystem of INAP is divided into three distinct layers that allow the establishment of information flows between different software components that compose them, in order to provide an ecosystem with a high interoperability degree among the different components as well as it evolution capability [6].

Figure 1 show the three layers. First, the services layer that covers the interfaces through users interact with the system. This layer includes the three main components of the ecosystem. Second, the static data management layer, whose aims is to manage the information shared among different components of the ecosystem and has a static nature due to it is not continuously updated. Finally, the infrastructure layer, whose aims is to provide supporting to the elements on top layers. For instance, this layer includes software components for the centralized user management or the server in charge of email management.

Noteworthy the Open Source philosophy followed by the ecosystem of INAP, following the strategy of the European Commission to use internally Open Source solutions [3; 4] or the commitment of the Government of Spain to foster knowledge and use of Free Software the National Center for the Application of Open Source Technologies (CENATIC) [19].

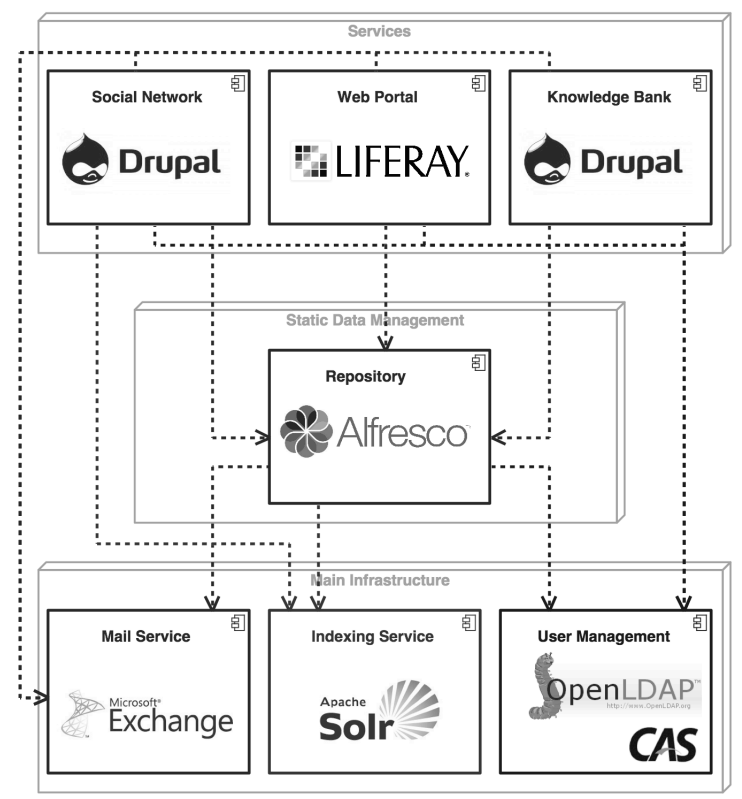

Figure 1. Architecture of the technological ecosystem of INAP [6] 


\subsection{Observatory for University Employability and Employment}

The Observatory for University Employability and Employment (Observatorio de Empleabilidad y Empleo Universitarios, OEEU in Spanish) (http://oeeu.org), is a technical unit that collects, produces, analyzes and disseminates information about employability and employment of university graduates in Spain, for which it has a network of researchers and technicians distributed throughout the country, organized into ten teams working in coordination with a single methodology led by the UNESCO Chair in University Management and Policy (based at the Technical University of Madrid) and the ongoing advice of an Expert Council, composed of national and international academics and university experts.

The main objective of OEEU is to become the epicenter of knowledge management about university employability and employment in Spain and supply the partial lack of analytical (academic, employability, etc.) unified to feed, strengthen and improve the systems and decision-making processes of the Universities Institutional Intelligence, likewise helping to create a "data-driven" culture of Institutional intelligence and knowledge management for decision making $[1 ; 16]$.

The network of researchers working for the Observatory has a range throughout the Spanish territory; they are grouping based on the autonomous territories and generating links between these groups and the Observatory, denominated collection centers and Information Analysis. The Observatory workflow combines three fundamental pillars: management, through the Management and Coordination Unit; a sound methodology that is based thanks to the work of an Expert Council; and a technological solution consists of different software components developed as it supports the work done by the Centers for Collection and Analysis of Information, establishing liaison with all Spanish universities and allow the collection of information from universities and graduates, nourishing the knowledge base on employability relative to graduates in Spain.

To support workflows, and processes information generated by the activity of the Observatory, it has opted for the creation, development and operation of an information system that is able to collect, store, process and exploit information and results produced [12]. This information system is not a monolithic entity that brings together various functionalities and features, but actually is shaped in different tools, applications and subsystems based on Open Source technologies that make up a multifaceted entity as an ecosystem where components or parts interact and cooperate for a common goal. Among the various components, systems and subsystems that make up the OEEU's information system, can be highlighted the following (Figure 2):

- $\quad$ Support layer for processes and information flows: This layer provides the mechanisms, interfaces and services needed for the proper integration of the subsystems. It is an intermediary entity that facilitates interoperability between components, decoupling information flows and logic of each of the subsystems, which simply focus on functionality and communicate with this entity.

- Data persistence layer: This layer is responsible for storing the information in a structured, accessible and in a standardized way, so that any system or application could be able to directly use and extract the contained information.
- System for information collection: it is responsible for collecting information from different sources, such as administrative records of the different universities involved in the Observatory activities and the Spanish graduates who complete the information of their universities, expressing their opinions on issues, their perception of their academic training, their skills and competencies, etc. These subsystems are described a little better below:

- Subsystem for collecting information from the Universities: This subsystem provides data collection procedures, methods and technical requirements for collecting properly the administrative information provided by the universities to the system.

- Subsystem for collecting information of graduates: this subsystem, based on administrative records provided by universities, creates customized questionnaires for each of the graduates registered in the system, allowing to record relevant information for Observatory purposes that can provide every graduate of the universities involved.

Besides these two subsystems, that are integrated into the solution currently deployed, there are other subsystems designed for integrating services through third party tools, external databases,

\section{etc. that would expand the ecosystem.}

Information analysis system: this system handles compute, organize and perform analysis on the information entered by students and universities in the system, leading to interim and final results for the Observatory that will be used in its reports, websites and public communications.

- Information reporting system: This system is responsible for filtering and present information appropriately to the various possible roles of users who can access the information of the Observatory (public access, participating universities, administrators, etc.)

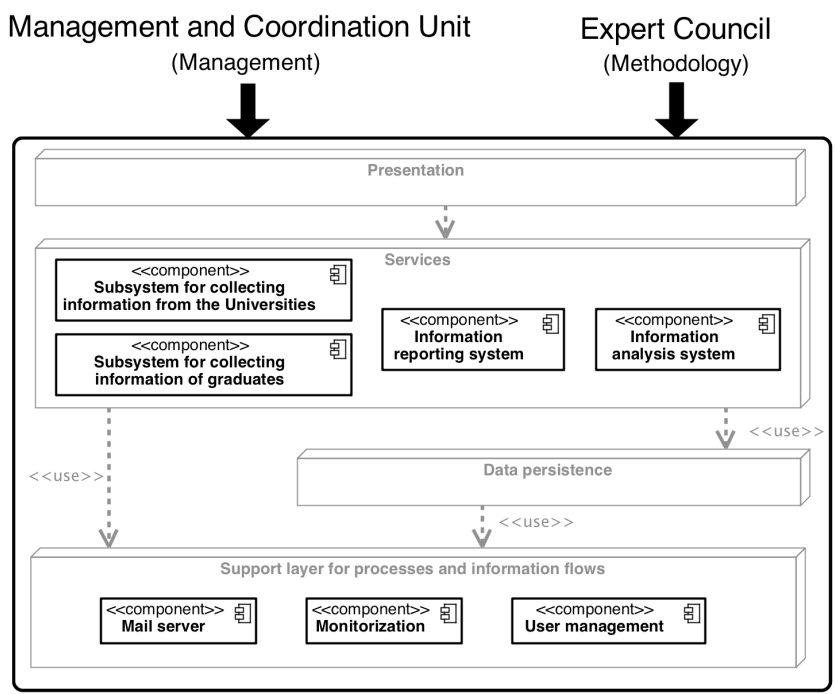

Figure 2. Architecture of the technological ecosystem for OEEU 


\section{RESULTS}

Issues related to the Knowledge Society include not only technological issues but should be accompanied by methodological elements that allow the optimization of resources related to knowledge. There is therefore a need for innovative models for knowledge management focused both staff and organizational development [11]. In this context arises the Suricata Model [18], an architectural proposal based on five layers ranging from the technological infrastructure that encompasses software, hardware and communications with a Open Source philosophy, to the personalized portal for knowledge worker, through which the user interacts with all layers of the architecture in a transparent way [9].

This model allows to develop methods and tools to support individuals involved in the processes of knowledge management, both from a personal and collective point of view, in order to promote increased productivity and innovation capacity in a context of knowledge management oriented to processes [18].

When analyzing the cases described in the previous section, has taken the Suricata Model as a basis for analyzing the different elements; on the one hand, the architecture of the INAP's technology ecosystem and secondly the technology ecosystem that sustains the OEEU.

First, the most basic layer of the Suricata architecture is the technological infrastructure, which aims to integrate the different software applications and facilitates their interoperability and integration. In the case of INAP, this layer is matched to the lower layer, the infrastructure layer, where interoperability between all components is facilitated through the centralized management of users through Lightweight Directory Access Protocol (LDAP) combined with Central Authentication Service (CAS). Regarding the case of the OEEU, this Suricata architecture layer can be likened to the support layer for processes and information flows as it acts in the same way that the INAP's infrastructure layer, providing the services and support to the other layers, coordinating networking and enabling cooperation among them.

The Suricata Model backs the architecture of the information in the technological infrastructure, so that this layer is responsible for storing digital artifacts, and allowing the content management. Both the ecosystem INAP as the OEEU have a data persistence layer in which all relevant information is handled for the knowledge management.

The third layer is the collaborative environment that enables collaboration among all the components of the architecture, ensuring the knowledge exchange. In the case of INAP's ecosystem, the top layer of the architecture, the service layer, would represent this layer so that some of the services included within have the same aim to facilitate the exchange of knowledge among members of Public Administration through the communities of practices and the knowledge bank. Regarding the OEEU's ecosystem this layer would be comparable to the layers that make possible the collection, analysis and reporting. These layers, that provide the services and cooperation for the knowledge exchange, that specifies the Suricata Architecture, but from a different point of view of the INAP case, since in this case the services are not directly related to other components and layers of the system, but they base their functionality in facilitating the information acquisition, knowledge extraction and knowledge exchange with the different actors that act as sources of information (universities and graduates), data analysts (OEEU) and consumers and disseminators information and knowledge (OEEU, universities, general public).
The penultimate layer of the Suricata Architecture focuses on support for the creation of virtual communities that allow the transaction of knowledge and not just the transmission thereof between different users [10]. Both ecosystems pose the transaction of knowledge as fundamental axis, while the focus varies on each. In the INAP this goal is evident by the aforementioned communities of practices. In the OEEU case, the knowledge transaction is performed, as also discussed above, through the combination of different services that allow collect, process, analyze, and transfer the collected knowledge organized and discovered by the ecosystem to generate new knowledge, through the inclusion of the human factor as an additional engine that could catalyze new knowledge through their interaction with knowledge as an analysts, observers, information consumer, trends discoverers and ultimately, as an entity that feeds -in a quasi-chaotic way from the point of view of a programmed and sufficiently open to interaction system- any system publicly available on the Internet today.

The last layer corresponds to the user interface through a customized environment that allows access to all layers of the architecture. In the case of INAP, there is a layer of access to other layers, but directly access the different elements of the service layer. However, in the case of OEEU, there is a last layer, called presentation layer, through which is provided a uniform user interface that enables the ecosystem perceived as a whole, the layer focuses on the visual appearance (through Information Visualization techniques) and using the interaction for knowledge analysis processes.

Therefore, it is possible to say that knowledge management cases analyzed, despite substantial differences, have common elements that are reflected when a comparison with the Suricata Model, so that they may be considered success stories from the point of view of utility and organization of knowledge in the public sphere, and also extrapolating those points common to other technological ecosystem focused on knowledge management in the context of public administration.

\section{CONCLUSIONS}

This article deals with how knowledge is managed from a technological standpoint in different public entities, such as public administration or university level institutions. For this, the paper presents several key points that put the reader in the context of the problem, showing various cases of technological ecosystems for knowledge managing, such as the ecosystem of the National Institute of Public Administration (INAP) and the Observatory for University Employability and Employment (OEEU) of the UNESCO Chair University Management and Policy based at the Technical University of Madrid. In these analysed case studies, are presented processes of generation, management and knowledge exploitation in different contexts and for different purposes in order to provide two different cases but with common characteristics and problems that provide to the reader an idea of how can be achieved the challenges of knowledge management presented, and facing its proposals with a model like the Suricata Architecture, enabling the comparison of how this architecture organizes knowledge management processes and how they do the cases presented. Based on that analysis and comparison, it is possible to determine some success factors in knowledge managing in the context of the public elements that share both case studies starting from a clear definition of the architecture to the incorporation of the human component as part of the system. 


\section{ACKNOWLEDGMENTS}

This research work is made within University of Salamanca PhD Programme on Education in the Knowledge Society scope and has been funded by the National Institution of Public Administration (INAP) and the Observatorio de Empleabilidad y Empleo Universitarios (OEEU).

Authors would like to thank their partners at Research Group in InterAction and eLearning (GRIAL) http://grial.usal.es and the Research Institute for Educational Sciences (IUCE) at the University of Salamanca http://iuce.usal.es for their contributions and support.

\section{REFERENCES}

[1] Alcolea Picazo, J. and Pavón De Paula, S., 2013. Los datos como recurso estratégico Libro Blanco Inteligencia Institucional en Universidades (pp. 17-43). Madrid, Spain: OCU (Oficina de Cooperación Universitaria).

[2] Arenilla Sáez, M., 2014. Conocimiento transformador y talento público. El caso del INAP. National Institute of Public Administration, Madrid, Spain.

[3] European Commission, 2009. European Union Public Licence (EUPL).

[4] European Commission Directorate-General for Informatics (Digit), 2011. Strategy for internal use of OSS at the EC.

[5] García-Holgado, A. and García-Peñalvo, F.J., 2013. The evolution of the technological ecosystems: an architectural proposal to enhancing learning processes. In Proceedings of the First International Conference on Technological Ecosystem for Enhancing Multiculturality (TEEM'13) (Salamanca, Spain, November 14-15, 2013) ACM, New York, NY, USA, 565-571.

http://dx.doi.org/10.1145/2536536.2536623

[6] García-Holgado, A. and García-Peñalvo, F.J., 2014. Architectural pattern for the definition of eLearning ecosystems based on Open Source developments. In Proceedings of 2014 International Symposium on Computers in Education (SIIE) (Logroño, La Rioja, Spain, November 12-14, 2014), J.L. Sierra-Rodríguez, J.M. Dodero-Beardo and D. Burgos Eds. Institute of Electrical and Electronics Engineers. HEEE Catalog Number CFP1486T-ART, 93-98. DOI= http://dx.doi.org/10.1109/SIIE.2014.7017711.

[7] García-Holgado, A., García-Peñalvo, F.J., HernándezGarcía, Á., and Llorens-Largo, F., 2015. Analysis and Improvement of Knowledge Management Processes in Organizations Using the Business Process Model Notation. In New Information and Communication Technologies for Knowledge Management in Organizations: 5th Global Innovation and Knowledge Academy Conference, GIKA 2015, Valencia, Spain, July 14-16, 2015, Proceedings, D. Palacios-Marqués, D.R. Soriano and K.H. Huarng Eds. Springer International Publishing.
[8] Hargreaves, A., 2003. Teaching in the knowledge society: Education in the age of insecurity. Teachers College Press.

[9] Marrero, S., Ocón, A., Galán, M., and Rubio, E., 2005. Methodology for the generation and maintenance of a "base of procedures" in process-oriented knowledge management strategy European University Information Systems (EUNIS).

[10] Marrero, S.R., Nelson, J.C., Galán, M., Ocón, A., and Rubio, E., 2005. Metodología para organizar, recuperar y compartir recursos de información y conocimiento en un centro $\mathrm{I}+\mathrm{D}+\mathrm{i}$ en la Plataforma Suricata.

[11] Meiler Rodríguez, M.C., 2014. Propuesta metodológica de envejecimiento activo basada en aprendizaje en red: Comunidad Seventi. In Centro de Innovación para la Sociedad de la Información - CICEI Universidad de Las Palmas de Gran Canaria, Las Palmas de Gran Canaria, 140.

[12] Michavila, F., Martín-González, M., Martínez, J.M., García-Peñalvo, F.J., and Cruz-Benito, J., 2015. Analyzing the employability and employment factors of graduate students in Spain: The OEEU Information System. In Proceedings of the Third International Conference on Technological Ecosystems for Enhancing Multiculturality (TEEM'15) (Porto, Portuga12015), ACM Inc.

[13] Natali, A.C.C. and Falbo, R., 2002. Knowledge management in software engineering environments. In Proceedings of the XVI Brazilian Symposium on Software Engineering (SBES'2002), 238-253.

[14] Nonaka, I. and Takeuchi, H., 1995. The knowledgecreating company: How Japanese companies create the dynamics of innovation. Oxford university press.

[15] Oecd, 2000. Knowledge Management in the Learning Society. OECD Publishing, Paris.

Oficina De Cooperación Universitaria. Ocu, 2013. Libro Blanco Inteligencia Institucional en Universidades Madrid: OCU.

[17] Rowley, J., 2007. The wisdom hierarchy: representations of the DIKW hierarchy. Journal of information science 33, 2, 163-180. DOI= http://dx.doi.org/10.1177/0165551506070706.

[18] Rubio, E., Ocón, A., Galán, M., Marrero, S., and Nelson, J.C., 2004. A personal and corporative processoriented knowledge manager: Suricata model European University Information Systems (EUNIS).

[19] Trejo Pulido, A., Domínguez Dorado, M., and Ramsamy, P., 2011. Open source software in public organisations of the Spanish government. 2011. CENATIC.

[20] Unesco, 2005. UNESCO World Report: Towards Knowledge Societies. UNESCO Publishing, Paris.

[21] Zeleny, M., 1987. Management support systems: Towards integrated knowledge. Human systems management 7, 59-70. 